EVIDENCE BASED PUBLIC HEALTH POLICY AND PRACTICE

\title{
How would schools step up public health measures to control spread of SARS?
}

\author{
A Lee, F F K Cheng, H Yuen, M Ho, and the Hong Kong Healthy Schools Support Group*
}

J Epidemiol Community Health 2003;57:945-949

The severe acute respiratory syndrome (SARS) is a rapidly progressive, and sometime fatal disease with more than 1800 patients in over a dozen countries in Asia, Europe, and North America (including the United States and Canada) within two months. On 12 March 2003, the World Health Organisation (WHO) issued a global alert about SARS so it became a global challenge. Strengthening the public health measures at schools would protect children as well as providing the students an opportunity to learn about infectious disease control through life event approach. The public health measures at schools include two important components: basic understanding of the disease so schools would put on high alert on caution cases, and the measures to improve environmental hygiene at schools and preventive measures to stop infectious disease transmission. This will help to empower the whole community the readiness to deal with other outbreaks in the future.

S evere acute respiratory syndrome (SARS) was diagnosed in more than 1800 patients in over a dozen countries in Asia, Europe, and North America (including the United States and Canada) within two months period (1 February and 31 March 2003). ${ }^{12}$ On 12 March, the World Health Organisation (WHO) issued a global alert about SARS, and on 14 March, the Center of Disease Control and Prevention (CDC) activated its emergency operation centre to support the response of the WHO to this global threat. Case clustering in Hong Kong and Canada makes it quite clear that SARS is an infectious disease. ${ }^{3-5}$ Epidemiological investigations and laboratory studies suggested that most patients with the disease meeting the criteria of SARS in Canada can be linked to a common potential causative agents. ${ }^{5}$ So far based on preliminary investigation, it seems that the syndrome may be attributable to a newly described respiratory viral pathogen, human metpnemovirus, to a novel coronavirus, or both. ${ }^{5-7}$

SARS has spread throughout the world because people with early symptoms can travel from one part of the world to another within half a day. Hong Kong is an important financial centre in the world and is also the heart of Asia, and therefore has rapid movement of population from different parts of the world so it is hard hit by SARS. SARS is now a global health hazard, and its high infectivity is alarming. Early recognition, prompt isolation, and appropriate treatment are the keys in combating the infection. Each school accommodates a substantial number of students and staff coming from different background so chance of cross infection of diseases especially by

*Grace Au, Suzanne Au, Vera Keung, Tracy Leung, Nancy Li, Amelia Lo, Phoebe Ng, Janet Ngan, Daimian Siu, Carol Suen, Grace Tso, Jasmine Wong, Vivian Wong, Winki Yuen. close contact, is very high. Therefore it is important for schools to have good public health measures to prevent infection.

\section{BASIC UNDERSTANDING OF SARS AND DEVELOPMENT OF MANAGEMENT FLOW CHART TO DEAL WITH CASES}

In response to the rapid outbreak of SARS, the public health measures to control spread of this infectious disease can be broadly divided into two aspects. Firstly, the school must have basic understanding of SARS including the presenting symptoms, who will be at risk, and the management guidelines in primary care. Then a management flow chart would be formulated for school setting. Secondly, the school needs to improve environmental hygiene at school and step up the measures to prevent direct transmission of the disease.

How would school workers be alerted of SARS cases? A recent article by CDC has described the clinical feature of SARS. ${ }^{8}$ The illness begins with fever $\left(>38.0^{\circ} \mathrm{C}\right.$ or $\left.>100.4^{\circ} \mathrm{F}\right)$. Fever is often high with chills and rigors, and might also be accompanied by other symptoms such as headache, malaise, and myalgia. Some might have minor respiratory symptoms such as a dry cough. The incubation period is generally two to seven days but some cases have been reported with an incubation period as long as 10 days. ${ }^{34}$ Of the 138 cases reported by the Chinese University of Hong Kong (CUHK), ${ }^{3}$ 10 cases reported by University of Hong Kong (HKU), ${ }^{4}$ and 10 cases in Canada 5 ; all patients had history of fever. Chills or rigor was reported by $73.2 \%$ of CUHK reported cases and $90 \%$ of HKU reported cases. Table 1 shows the presenting characteristics of the cases of the three reports. ${ }^{3-5}$

In the primary care setting, it is difficult to differentiate community acquired pneumonia, atypical pneumonia from simple flu cases during the initial phase. However, in view of recent outbreaks, the Hong Kong Hospital Authority has issued guidelines for primary care doctors (http:// www.ha.org.hk/sars/guidelines/index.html) to alert them for the following clinical manifestations ${ }^{9}$ :

- Fever $>38^{\circ} \mathrm{C}$, or history of fever over the past two days, AND

- One or more respiratory symptoms including cough, shortness of breath, difficulty in breathing, AND

- At least two of the following:

- Chills any time in the past two days

- New or increased cough

- (Generalised) malaise

- Typical physical signs of consolidation

- Close contact within the past two weeks with a person who has been diagnosed with SARS. Close contact means having cared for, having lived with, or having had direct 


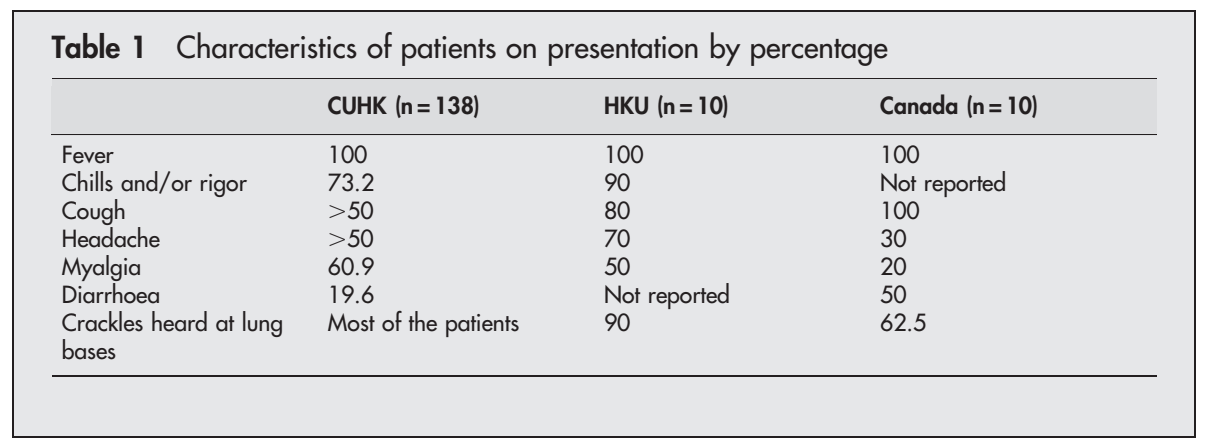

contact with respiratory secretions and body fluids of a person with SARS

- Associated symptoms such as headache, myalgia/muscular stiffness, pleuritic chest pain, loss of appetite, confusion, rash, and diarrhoea

(If chest radiograph shows lobar consolidation or the pathogen is already known, the case can be excluded)

The guidelines advise primary care doctors to refer those patients with fever $>38^{\circ} \mathrm{C}$ and history of contact to hospital for further management if they develop new onset of pulmonary infiltrate (clinical investigation or radiography, or both) AND with symptoms of shortness of breath or cough AND patient has been exposed with pneumonia in the previous two weeks. Primary care physicians are also advised to refer those patients with fever $>38^{\circ} \mathrm{C}$ and new onset of pulmonary infiltrate (clinical investigation or radiography, or both) but NO history of contact to hospital for further management if patients have shortness of breath or cough AND no symptomatic response to standard treatment including a $\beta$-lactam (penicillin and cephalosporin groups) and coverage for atypical pneumonia (a fluoroquinolone, tetracyclines, or a macrolide) after two days of treatment.

Based on available clinical information on SARS, ${ }^{2-4} 8$ and the hospital authority guidelines for primary care physicians, ${ }^{910}$ the Centre for Health Education and Health Promotion of the Chinese University of Hong Kong (CHEP) has designed a management flowchart to assist the schools to triage students with suspicion of possible SARS cases (fig 1). CHEP has also designed the health assessment form for students and staff (appendix 1) in recording the warning symptoms of SARS and also the history of exposure. Based on the information recorded on the health assessment form, the school teachers would then refer to the management flowchart for appropriate action.

\section{IMPROVE ENVIRONMENTAL HYGIENE AT SCHOOLS (PREVENT INDIRECT TRANSMISSION)}

Improving environmental hygiene at school is an important step in blocking and cutting out the route of transmitting disease. The Centre for Health Education and Health Promotion launched the "Healthy Schools" programme in Hong Kong in 1998 and practical guidelines have already been established for schools to improve environmental hygiene and prevention of direct transmission of infectious disease to meet the standards of being a "Healthy School". ${ }^{11}{ }^{12}$ Schools have been advised to pay attention to the following points for improvement of the environmental hygiene:

- Provide a safe water supply system

- Provide appropriate hand cleaning facilities

- Have proper sewage and waste management systems

- Keep lavatories clean

- Provide good lighting and ventilation systems
- Provide meals (such as snack station and canteen) that meet the standards set by the Department of Health

\section{In healthy school practice, the school should}

- Ensure good indoor air ventilation

- Maintain a good air conditioning system and clean filter of air conditioning frequently

- Keep windows opened and use exhaust fan in crowded environments to improve ventilation

- Keep windows opened during class for good ventilation

- Keep premises clean and halt the growth of rats, mosquitoes, and bugs

- Always keep the lid on rubbish bins and wash the bin at least once a day

- Keep floors, ceilings, and walls clean and make sure drainages and water pipes are clear of obstacles

- Use washable carpets and wash them regularly

- To halt the growth of mosquitoes, clear stagnant water promptly and replace water inside containers at least once a week

- Keep lavatories clean

- Clean the toilet with household bleach diluted 99 times at least once a day and wash with water afterwards and wipe it dry*

- Maintain proper flushing operation of toilets

- Lavatories should be supplied with cleansing liquid, toilet paper, paper towels or hand drying machines.

- Towels should not be shared and have to be washed regularly (can soak towels with diluted household bleach for 30 minutes before washing with water)*

- Clean furniture and toys regularly

- Clean furniture and toys with diluted household bleach at least once a day (furniture that cannot be washed with water can be wiped with towels that have been soaked completely in household bleach)*

- Washable furniture and toys should be washed once a day and be soaked in 1:99 household bleach for 30 minutes before washing*

- Objects that are contaminated by vomitus and excretions should be handled properly

*The frequency of cleaning depends on the use of the facilities and school circumstances. The above recommendation is only a minimal requirement. 


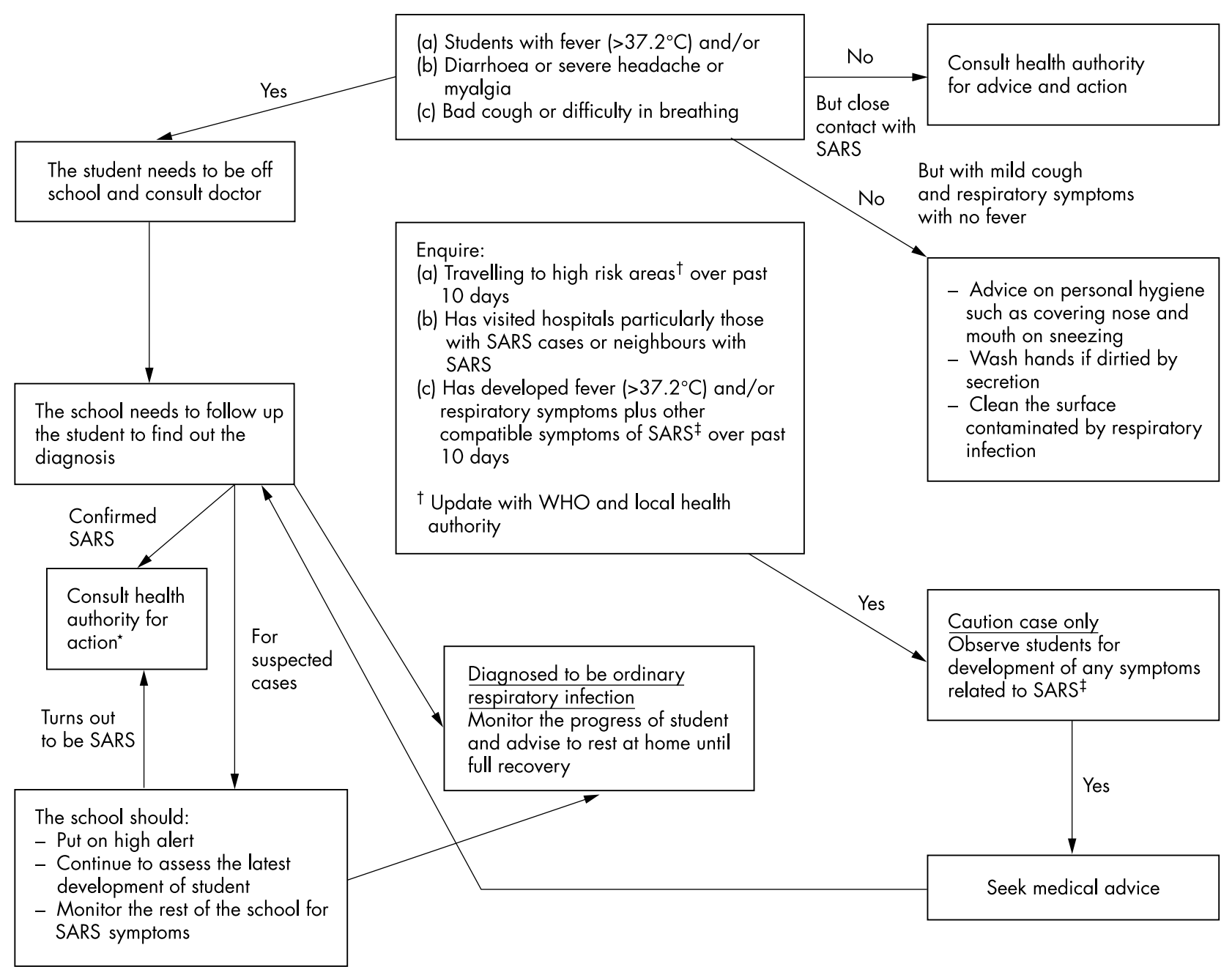

* In Hong Kong, the school will be closed for 10 days and staff and students will be under surveillance. School will be disinfected.
‡In addition to fever and respiratory symptoms, SARS may be associated with headache, muscular stiffness, loss of appetite, malasie, confusion, and diarrhoea

Figure 1 Management flowchart for school teachers during epidemics of SARS.

- Contaminated surfaces should be cleaned immediately with 1:49 diluted household bleach (mix 1 portion of bleach with 49 portions of water).

- The school should avoid cleaning student's personal belongings that are contaminated. These contaminated items should be put into plastic bags and be collected by parents.

\section{PREVENTING DIRECT TRANSMISSION}

Improving environmental hygiene at school and vaccination can only prevent the outbreak of certain infectious diseases. The root of transmission is the patient him/herself. Early detection and isolation of infected persons can reduce the chance of spreading infectious diseases. To achieve this goal, the school should set and practice a policy that governs the handlings and controlling of the transmission root.

\section{Early detection of infection}

The earlier a case of infection is detected, the lower the chance of transmission. The school should therefore be vigilant. Apart from relying on the ordinary daily observations by parents, the school should also rely on the observations provided by its staff during morning assemblies or other times to help detect early infections.

School should inform and advise parents to bring their children to seek early medical advice if they suspect their children of having signs and symptoms of certain infectious diseases. A previous section has discussed how the school should be alerted for potential SARS cases. The flowchart (fig 1) would facilitate the school to handle students with potential risk of developing SARS.

\section{Investigate absentees and report on cases of infectious diseases}

Keeping a proper record of sick leave for individual students and staff at school would help to alert the health authority of suspected outbreak of infectious disease. To help investigation and control of infectious diseases, the school should obtain consent from parents/guardians to provide immediately notification to the health authority. The school should contact the health authority for investigations and follow up activities if an excessive amount of absentees experience similar symptoms. 


\section{Students with infectious diseases should be excluded from school}

To stop the spread of infectious diseases, infected students should take rest at home and be excluded from school. The duration of exclusion depends on the type of infectious disease. In Hong Kong, the Department of Health has made recommendation on duration of exclusion from schools for different types of illnesses (http://www.info.gov.hk/dh/disease/guide). The school should take appropriate actions with reference to the suggestions given by the local health authority.

\section{The returning to school of recovered students}

Decisions on students returning to schools after recovering from infectious diseases bear greater significance than the decision to exclude. Pathogens may still be present inside the bodies of students who have just recovered so they may infect others in school if the infectious period still persists. Therefore the school is recommended to admit return only upon doctors' recommendation.

\section{Carry out disinfections during the outbreak of infectious diseases}

The purpose of disinfecting is to kill off the pathogen. Thorough incineration, boiling, ultraviolet light, or other chemical methods to handle excretions and discharges that can possibly spread the diseases should be undertaken. With regard to common infectious diseases, the practical guidelines developed by CHEP advise the schools to carry out the following disinfecting tasks during an outbreak of infectious diseases ${ }^{12}$ :

- Use household bleach diluted 49 times (mix a portion of bleach with 49 portions of water) to disinfect furniture, floors, and lavatories. Wash with water and wipe the objects dry after 30 minutes

- Used paper towels should be handled properly. Used paper towels should be disposed of immediately and be put in a rubbish bin with lid. Used towels should be immersed into household bleach diluted 49 times for 30 minutes before washing with water.

- Surfaces contaminated with vomit, excretions, discharges, or blood should be wiped with towels that have been soaked with household bleach diluted 49 times and be washed with water after 30 minutes (at best, towels should be disposed of immediately after use).

- Use household bleach diluted 49 times to disinfect toilet and toilet seat. Wash with water after 30 minutes.

\section{Education on hygiene}

The control and prevention of the outbreak of infectious diseases is not the only aim of the school's control and prevention scheme. School can also use relevant experiences learnt from the job to educate students. On the one hand students are protected from infection, and on the other hand they can learn to avoid passing the infections to others.

The key points of education include:

- Educate correct knowledge on prevention and control of infectious disease

- Help to develop the correct behaviour on health and prevention of infectious disease. Examples include washing hands before meals and after going to the toilet, or when affected by respiratory secretions; and not sharing towels, disposable towels and tissues; cover nose and mouth when sneezing or coughing, and dispose of respiratory secretions with care
- Educate students to maintain good environmental hygiene at school, such as not dropping litter, which helps to decrease spreading of disease

- Strengthen body immunity through balanced diet and adequate fluid intake, regular exercise and adequate sleep, reducing stress, and avoid smoking

- When students, teachers, or staff are suspected of suffering from respiratory tract infections, they are advised to seek early medical treatment and rest at home whenever necessary

- Increase the transparency of all facilities for preventing and controlling infectious diseases at school to enable students to obtain a thorough understanding and encourage students to accept and participate

- Promote education for parents and obtain cooperation from parents

The Chinese University of Hong Kong launched the "Schools Against SARS" educational campaign with the aim to better equip school principals, teachers, and students of secondary and primary schools to fight against SARS. The campaign has drawn upon the medical expertise and the experience of the Chinese University's Medical Faculty pooling experts from CHEP, School of Nursing and also frontline medical clinicians who have extensive first hand experience in dealing with SARS. An educational video was produced with practical advice on preventive measures against SARS in the school setting, and promoting support among Hong Kong people. The video is now available on a web site (http://www.hkedcity.net/project/cuhk sars/index e.phtml). Pamphlets and videos have been made available to 1000000 primary and secondary school students. The educational video has adopted a school based approach to put hygiene education into practice so staff, students, and parents can be equipped with the skills in prevention.

\section{CONCLUSION}

SARS has already become a global challenge, and its infectivity is alarming. It is unrealistic to hope that the disease will burn itself out very soon. ${ }^{2}$ The key message is caution not panic. Schools have the dual responsibility of protecting the welfare of students and provision of education. Strengthening the public health measures at schools would protect our children as well as providing the students an opportunity to learn about infectious disease control through life event approach. This will help to empower the whole community with the readiness to deal with another outbreaks in future.

\section{Authors' affiliations}

A Lee, F F K Cheng, H Yuen, M Ho, Centre for Health Education and Health Promotion, School of Public Health, The Chinese University of Hong Kong, Hong Kong

Correspondence to: Professor A Lee, Centre for Health Education and Health Promotion, The School of Public Health, The Chinese University of Hong Kong, 4th Floor, School of Public Health, Prince of Wales Hospital, Shatin, NT, Hong Kong; alee@cuhk.edu.hk

Accepted for publication 29 September 2003

\section{APPENDIX 1 HEALTH ASSESSMENT FORM Part A-Personal health}

- Do you have any fever $\left(>38^{\circ} \mathrm{C}\right)$ ?

- Do you have any diarrhoea, or severe headache or myalgia now ?

- Do you have any cough especially dry cough lasting for more than a day? 
- Do you have any difficulty in breathing?

- Do you have any runny nose and/or occasional cough?

- During the past 10 days, have you had fever $\left(>38^{\circ} \mathrm{C}\right)$ and dry cough lasting for more than a day?

\section{Part B-Social history}

- Have you had any close contact with any person who has been diagnosed with SARS?

- Have you been away over the past 10 days? If yes, please state where did you go? Update with WHO for high risk areas (Mainland China, Hong Kong, Singapore, Vietnam, Thailand, Canada).

- Have you visited any hospital over the past 10 days especially those hospitals or wards with SARS cases?

- Do you have any neighbours been diagnosed with SARS?

\section{REFERENCES}

1 Gerberding JL. Faster but fast enough? Responding to the epidemics of severe acute respiratory syndrome. N Engl J Med 2003 hitp:// www.nejm.org (accessed 2 Apr 2003).

2 Drazen JM. Case custers of the severe acute respiratory syndrome. N Engl J Med 2003 http://www.nejm.org (accessed 31 Mar 2003).
3 Lee $\mathrm{N}$, Hui D, Wu A, et al. A major outbreak of severe acute respiratory syndrome in Hong Kong. N Engl J Med 2003 http://www. nejm.org laccessed 7 Apr 2003)

4 Tsang KW, Ho PL, Ooi GC, et al. A cluster of cases of severe acute respiratory syndrome in Hong Kong. N Engl J Med 2003 http://www.nejm.org (accessed 31 Mar 2003).

5 Poutanen SM, Low DE, Henry B, et al. Identification of severe acute respiratory syndrome in Canada. N Engl J Med 2003 http://www.nejm.org (accessed 31 Mar 2003).

6 Drosten C, Gunther S, Presier W, et al. Identification of a novel coronavirus in patients with severe respiratory syndrome. N Engl J Med $2003 \mathrm{http}: / /$ www.nejm.org (accessed 10 Apr 2003).

7 Ksiazek TG, Erdman D, Goldsmith C, et al. A novel coronavirus assoicated with severe respiratory syndrome. N Eng J Med 2003 http://www.nejm.org (accessed 10 Apr 2003).

8 CDC. Preliminary clinical description of severe acute respiratory syndrome. MMWR 2003;52:255-6. (http://www/cdc.gov/mmwr)

9 Hong Kong Hospital Authority. Clinical guidelines for SARS; 2003. (http:// www.ha.org.hk/sars/guidelines/index.html)

10 The Hong Kong Hospital Authority Working Group on SARS and Central Committee on Infection Control. Guideline on management of severe acute respiratory syndrome. Lancet published online 8 Sep 2003. http:// image. thelancet.com. extras $/ 03 \mathrm{~cm}+89$ web.pdf

11 Lee A, Lee SH, Tsang KK, et al. A comprehensive "Healthy Schools Programme" to promote school health: The Hong Kong experience in joining the efforts of health and education sectors. J Epidemiol Community Health 2003:573:174-7.

12 Lee A, ed. Hong Kong Healthy Schools award scheme: practical guide to the Health Promoting School. Hong Kong: Centre for Health Education and Health Promotion, School of Public Health, The Chinese University of Hong Kong, 2002. 